\title{
Determinación y propuesta de una investigación acción participativa en el Colegio Enrique Olaya Herrera
}

\author{
Jonathan Heiler Hernández Prieto \\ dqu_jhernandez009@pedagogica.edu.co \\ Universidad Pedagógica Nacional
}

\section{Resumen}

El artículo es resultado de un estudio que buscó establecer la conveniencia en aplicar la Investigación-Acción Participativa (IAP) en el aula ciencias naturales del Colegio Enrique Olaya Herrera (CEOH) IED, además si el educador y estudiantes conocían la IAP por medio tres instrumentos de elaboración propia. El diseño metodológico consistió en hacer una propuesta inicial de actividades concatenadas de agricultura urbana, plantas medicinales y suelos con enfoque IAP que podría ser ejecutada y desarrollada a futuro. De otra parte, el segundo semestre del 2013 se realizó la observación y registro de clases en un diario de campo; se aplicó una encuesta a estudiantes de grado noveno de educación básica y también una entrevista a una profesora de ciencias naturales del CEOH IED, ubicado en Bogotá D.C., Colombia. Cabe mencionar que se implementó una investigación cualitativa dirigida que servirá de apoyo a nuevas investigaciones en la enseñanza de las Ciencias Naturales con enfoque IAP.

\section{Palabras Clave}

IAP (Investigación-Acción Participativa), Enseñanza de las Ciencias Naturales, Diario de Campo, Encuesta y Entrevista.

Recibido 16/05/2020-Aceptado 1/06/2020 


\title{
Determination and proposal of a research participatory action in the Enrique Olaya Herrera school
}

\begin{abstract}
The article is the result of a study that sought to establish the convenience in applying Participatory Action Research (IAP) in the natural science classroom of the Enrique Olaya Herrera College $(\mathrm{CEOH})$ IED, also if the educator and students knew the IAP through three instruments of own elaboration. The methodological design consisted of making an initial proposal of concatenated activities of urban agriculture, medicinal plants and soils with an IAP approach that could be executed and developed in the future. On the other hand, in the second semester of 2013, the observation and recording of classes was made in a field newspaper; A survey was applied to ninth grade students of basic education and also an interview with a professor of natural sciences at CEOH IED, located in Bogotá D.C., Colombia. It is worth mentioning that a directed qualitative research was implemented that will support new research in the teaching of Natural Sciences with an IAP focus.
\end{abstract}

\section{Keywords}

P.A.R. (Participatory-Action Research), Teaching of Natural Sciences, Field Diary, Survey and Interview. 


\section{Determinação e proposta de uma investigação-ação participativa na escola enrique de Olaya Herrera}

\section{Resumo}

O artigo é o resultado de um estudo que buscou estabelecer a conveniência de aplicar a Pesquisa Participativa em Ação (IAP) na sala de ciências naturais do IED da Enrique Olaya Herrera College $(\mathrm{CEOH})$, também se o educador e os alunos conhecessem o IAP através de três instrumentos de elaboração própria. $\mathrm{O}$ desenho metodológico consistiu em fazer uma proposta inicial de atividades concatenadas da agricultura urbana, plantas medicinais e solos com uma abordagem de PIA que poderia ser executada e desenvolvida no futuro. Por outro lado, no segundo semestre de 2013, a observação e gravação das aulas foi realizada em um jornal de campo; uma pesquisa foi aplicada a alunos do nono ano do ensino fundamental e também uma entrevista com um professor de ciências naturais no CEOH IED, localizado em Bogotá, na Colômbia. Vale ressaltar que foi implementada uma pesquisa qualitativa direcionada que apoiará novas pesquisas no ensino de Ciências Naturais com foco no IAP.

\section{Palavras-chave}

PAP (Pesquisa-Ação Participativa), Ensino de Ciências Naturais, Diário de Campo, Pesquisa e Entrevista. 


\section{Introducción}

La Investigación-Acción Participativa (IAP) puede ser definida como:

Un método de investigación y aprendizaje colectivo de la realidad, basado en un análisis crítico con la participación activa de los grupos implicados, que se orienta a estimular la práctica transformadora y el cambio social. El método de la Investigación-Acción Participativa (IAP) combina dos procesos, el de conocer y el de actuar, implicando en ambos a la población cuya realidad se aborda. Al igual que otros enfoques participativos, la IAP proporciona a las comunidades y a las agencias de desarrollo un método para analizar y comprender mejor la realidad de la población (sus problemas, necesidades, capacidades, recursos), y les permite planificar acciones y medidas para transformarla y mejorarla. Es un proceso que combina la teoría y la praxis, y que posibilita el aprendizaje, la toma de conciencia crítica de la población sobre su realidad, su empoderamiento, el refuerzo y ampliación de sus redes sociales, su movilización colectiva y su acción transformadora (Eizagirre, M. \& Zabala, N. 2000).

Del mismo modo, otro punto de vista de la IAP la define como:

Un método de estudio y acción que busca obtener resultados fiables y útiles para mejorar situaciones colectivas, basando la investigación en la participación de los propios colectivos a investigar. Que así pasan de ser "objeto" de estudio a sujeto protagonista de la investigación, controlando e interactuando a lo largo del proceso investigador (diseño, fases, devolución, acciones, propuestas, entre otros) y necesitando una implicación y convivencia del investigador externo en la comunidad a estudiar (Nistal, T. 2007, p. 6).

Así, la IAP es una rama de la investigación social que puede ser empleada junto al área de ciencias naturales, en la cual los profesores se encuentran en constante capacitación y actualización sobre los conocimientos que son proporcionados por la comunidad científica con el fin de aplicarlos en contexto. De este modo, propuestas de IAP se pueden llevar a las comunidades de instituciones educativas para solucionar problemáticas cotidianas. En consecuencia, es importante resaltar que en la IAP existen componentes muy fuertes, como lo son: las condiciones sociales e históricas de una comunidad y de un territorio. Asimismo, en los últimos años han aumentado la cantidad de estudios de investigación y bagaje sobre el estilo investigativo IAP junto a la enseñanza de las ciencias naturales. Por ello, se esboza su determinación y una futura propuesta con una secuencia de actividades (Moncayo, 2015). 
De tal manera, que la IAP en las ciencias naturales permite construir nuevo conocimiento social y científico susceptible de ser convertido en ejes temáticos en el aprendizaje, evidentemente busca atacar problemáticas locales que puedan ser solucionadas a través de propuestas innovadoras desde el aula con acompañamiento de la comunidad educativa y afectada por la problemática (Miranda, 2008)

Navarro (2005) menciona que la educación basada en contenidos programáticos hace que los profesores estén alejados de los intereses y vivencias de las comunidades. Entonces, los docentes dejan a un lado la investigación social, la cual tiene un impacto educativo porque se adquiere conocimiento objetivo, se aprende a analizar los problemas comunitarios conjuntamente, se debate o delibera las diversas visiones y enfoques para implementar acciones concretas de mejora a dichos problemas, en pocas palabras, el aprendizaje en particular de las ciencias naturales debe ir a la par de la investigación para generar un aprendizaje colectivo.

Por tanto, este proyecto tuvo como objetivo determinar si se desarrolla la IAP junto a la enseñanza de las ciencias naturales del Colegio Enrique Olaya Herrera I.E.D. (CEOH IED) de grado noveno de secundaria. Para implementarlo se apoyó en: un referente teórico sobre la IAP, una metodología basada en una investigación cualitativa dirigida y los instrumentos implementados a como el diario de campo, la entrevista y la encuesta. El análisis de la investigación se realizó con una triangulación entre los instrumentos de la metodología y los resultados son presentados en tablas y gráficas.

Aparte se deja planteada para etapas posteriores de la investigación, una secuencia de actividades sencilla como ejemplo donde se puede usar la metodología IAP junto a la enseñanza de las ciencias naturales sobre agricultura urbana, plantas medicinales y suelos asociadas a experimentos de laboratorio. Por lo tanto, existen procesos en los que se debe contrastar la teoría con la experimentación a través de hipótesis, un análisis crítico, confrontar los resultados y para finalizar plantear nuevos problemas para obtener procesos válidos y confiables (Borda y Erazo, 2010). 
Finalmente, se encuentran las conclusiones, bibliografía y los respectivos anexos correspondientes a los instrumentos y la secuencia de actividades.

\section{Metodología}

Los instrumentos de la presente investigación se muestran de manera general en el siguiente esquema. Este esquema tiene algunas etapas en las cuales se determina la implementación de la IAP asociada a la enseñanza de las ciencias naturales, lo que permite construir una investigación cualitativa dirigida paso a paso al tener en cuenta los resultados y análisis de los mismos.

Esquema 1: Etapas del proceso investigativo.

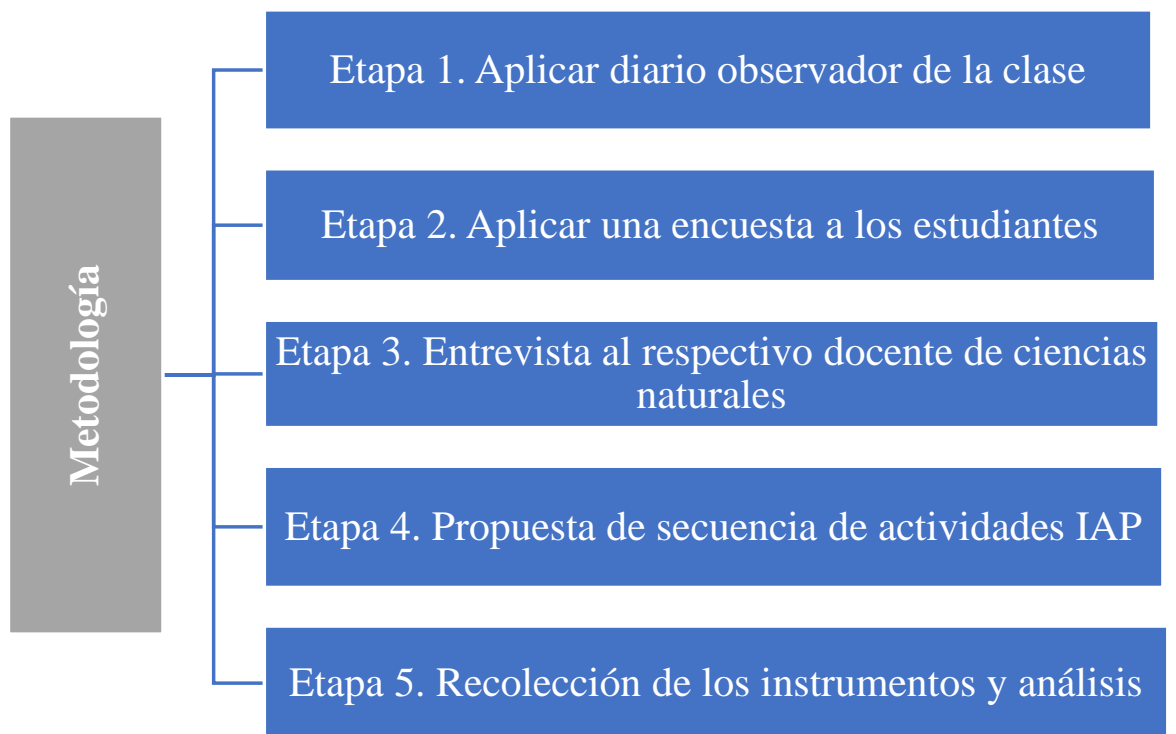

Fuente: Elaboración propia 


\section{Resultados y análisis}

\section{Etapa 1}

Cuadro 1: Resultados del diario de campo

\begin{tabular}{|c|c|c|}
\hline Descripción & Interpretación & $\begin{array}{l}\text { Reflexión y puntos de intervención } \\
\text { para la acción }\end{array}$ \\
\hline $\begin{array}{l}\text { La actividad de Clasificación } \\
\text { Taxonómica, fue dirigida por la } \\
\text { profesora titular con la ayuda del } \\
\text { practicante. El objetivo de la } \\
\text { actividad era desarrollar } \\
\text { habilidades y destrezas para } \\
\text { determinar taxonómicamente } \\
\text { seres vivos a partir de } \\
\text { observaciones directas. Con } \\
\text { anterioridad, se había pedido } \\
\text { algunos implementos como } \\
\text { guantes, lupa, frascos, etc. } \\
\text { Posteriormente, se seleccionaron } \\
\text { tres sitios en zonas distintas del } \\
\text { colegio, donde se ponía en } \\
\text { contacto a los estudiantes con su } \\
\text { entorno, seleccionaban especies } \\
\text { y las clasificaban } \\
\text { taxonómicamente. Finalmente, } \\
\text { realizaban un escrito en forma de } \\
\text { observación con una guía de } \\
\text { apoyo para los estudiantes. } \\
\text { Después, se alcanzó el acuerdo } \\
\text { de exponer la experiencia por } \\
\text { grupos en clase. }\end{array}$ & $\begin{array}{l}\text { La actividad fue bien dirigida } \\
\text { porque los estudiantes tomaban } \\
\text { observaciones, les gustaba } \\
\text { interactuar fuera del aula, } \\
\text { muchos tuvieron repugnancia a } \\
\text { los insectos, reconocieron } \\
\text { lugares que no conocían en el } \\
\text { colegio. También, lograron } \\
\text { cumplir y acatar los criterios } \\
\text { establecidos por la profesora } \\
\text { titular y el practicante, en las } \\
\text { exposiciones de los organismos y } \\
\text { especies debido a que se acentuó } \\
\text { más la reflexión sobre esta } \\
\text { actividad. }\end{array}$ & $\begin{array}{l}\text { Los estudiantes de grado noveno son } \\
\text { manejables. Las actividades prácticas } \\
\text { favorecen mucho el interés en las ciencias } \\
\text { naturales, puede que este tipo de } \\
\text { actividades fortalezca sus conocimientos } \\
\text { y su forma de pensar hacia las ciencias. } \\
\text { Tal vez, estas actividades articuladas a la } \\
\text { IAP logren un proceso de enseñanza y } \\
\text { aprendizaje más completo. }\end{array}$ \\
\hline
\end{tabular}

Formato Tomado de: (Muñoz, J. et. al., 2005, p.169). Fuente: Elaboración propia

Fecha: 29 de Agosto de 2013

Hora: 8-10 am

Episodio o Situación: Clasificación Taxonómica

Participantes: Profesora Titular y Docente Practicante Jonathan Hernández Prieto

Lugar: Colegio Enrique Olaya Herrera I.E.D. 
Según Téllez (2007) y Muñoz (2005) sobre esbozan que:

El diario de campo se emplea para escribir apuntes continuos de una investigación, de igual manera este diario de campo debe ser tanto un lente de observación para descripciones objetivadas como también un espejo de reflexión y autocritica para el investigador (2007, p. 178)

Este formato de diario de campo está basado en un ejemplo del libro de José Muñoz; este autor también menciona que el hecho de escribir un diario de campo apoya a reformar las fases de conciencia e interpretación, lo que le permite al investigador instaurar relaciones, ahondar en conceptos y sobre todo vincular la experiencia vivida diariamente tanto en el aula como en otros espacios de trabajo pedagógico (2005, p. 165).

En el diario de campo, empleado en esta investigación se analiza que la profesora encargada manejaba didáctica en sus clases a través de guías elaboradas previamente en la planeación, las cuales eran solucionadas por los estudiantes de manera individual, en parejas o en grupos. No obstante, si se vincula este tipo de actividades con la metodología IAP puede enriquecer los procesos de aprendizaje.

\section{Etapa 2}

En la encuesta aplicada a los estudiantes se presentan los siguientes resultados y un análisis de las preguntas 1 hasta la 8 , con el fin de mostrar cómo se cumplió el objetivo general de esta investigación.

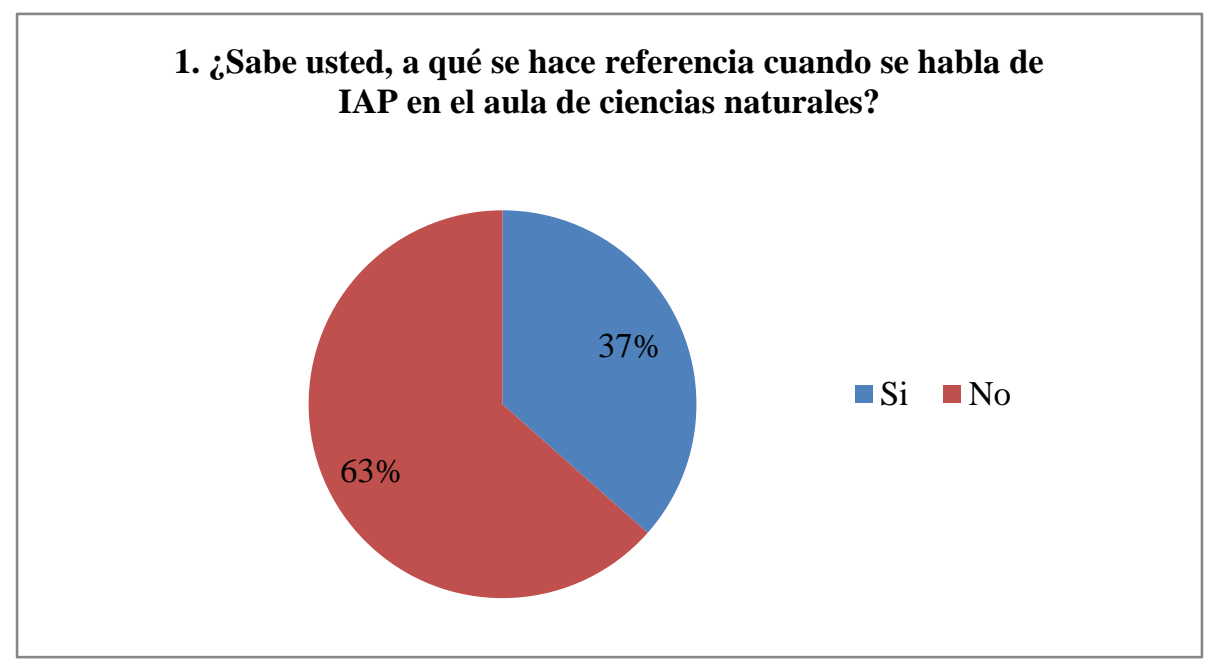

Figura 1: Pregunta 1. Fuente: Elaboración propia 


\begin{tabular}{lcc} 
Tabla 1 & \\
\hline Pregunta 1 de la encuesta. & Porcentaje y cantidad de estudiantes \\
\hline Respuesta & SI & NO \\
Cantidad de Estudiantes & 19 & 33 \\
Porcentaje \% & 37 & 63
\end{tabular}

Fuente: Elaboración propia

En esta pregunta la moda entre las personas encuestadas fue NO, es representativa con un $63 \%$ lo cual indica que los estudiantes no tenían alguna idea referente a la IAP.

La pregunta 2 ¿Qué es IAP en el aula de ciencias naturales? Al analizar las respuestas a esta pregunta abierta se encontró separada la Investigación-Acción-Participación por parte de los estudiantes: en el caso de la Investigación, la categorizaban como la práctica del aula, como experimentos proyectos y propuestas de estudiantes para la clase de ciencias naturales, también la asociaban a la explicación o la exposición, donde se analiza y se formulan preguntas y la última categoría es asociarla a alternativas de enseñanza y aprendizaje de las ciencias naturales; la acción es relacionada con dar a conocer lo realizado, el aprendizaje de forma no teórica en el aula de ciencias naturales, sino a través de la indagación, elaboración y aprendizaje de una temática específica; y para terminar, la Participación que estaba relacionada con compartir el conocimiento científico como también participar en las actividades del aula e incluir en estos procesos a las comunidades aledañas.

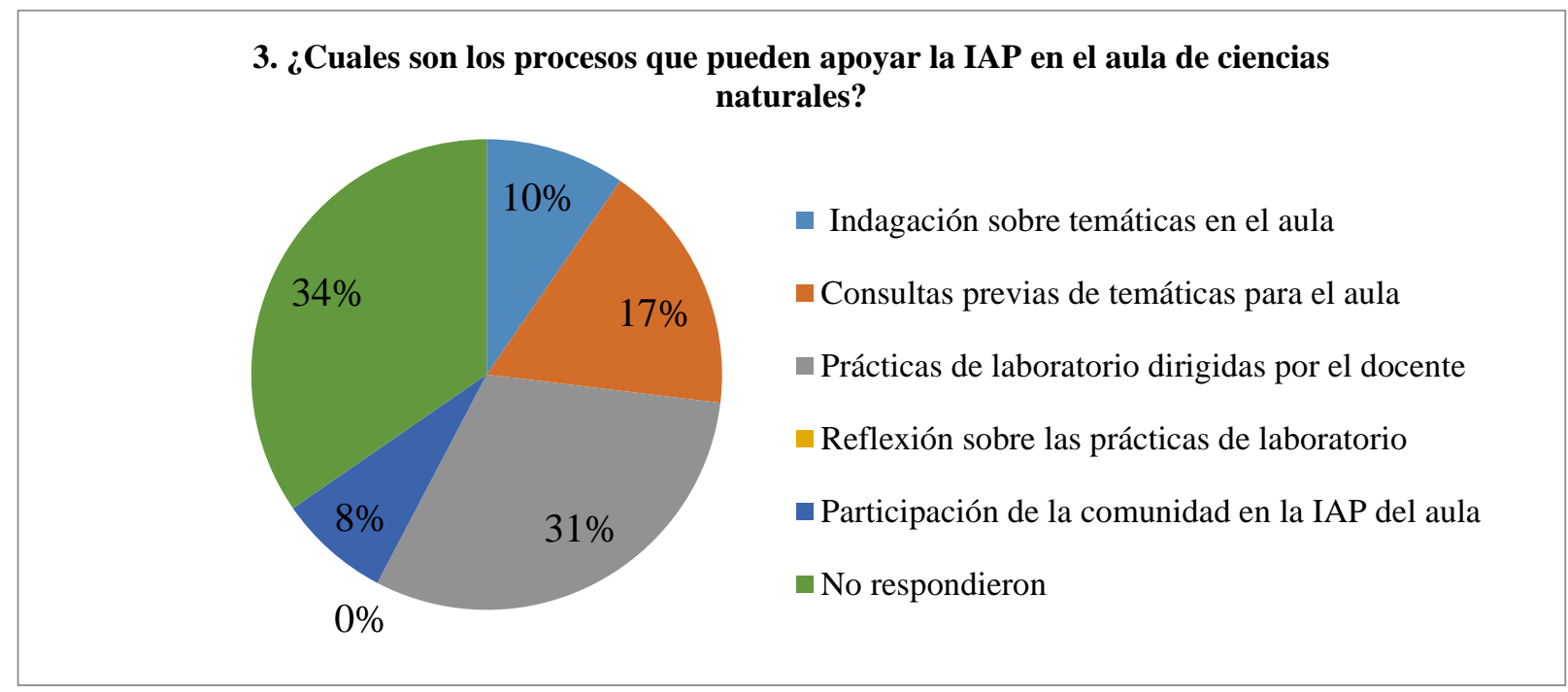

Figura 2: Pregunta 3. Fuente: Elaboración propia 
Tabla 2

Pregunta 3 de la encuesta. Porcentaje y cantidad de estudiantes

CARACTERÍSTICAS

Indagación sobre temáticas en el aula

Consultas previas de temáticas para el aula

Prácticas de laboratorio dirigidas por el docente

Reflexión sobre las prácticas de laboratorio

Participación de la comunidad en la IAP del aula

No respondieron
CANTIDAD DE PERSONAS

Fuente: Elaboración propia

Según la percepción de los estudiantes el proceso que puede apoyar la IAP son las prácticas de laboratorio con un $31 \%$, esta es la tendencia. No obstante, es poco representativa debido a que su porcentaje no sobrepasa la mitad de los estudiantes. En las respuestas abiertas se encuentra que la mayoría explicaban sobre sus consultas previas antes realizar la práctica de experimental, algunos escribían que la investigación empieza por la consulta y otros exponían que se investiga antes de que se procese la teoría.

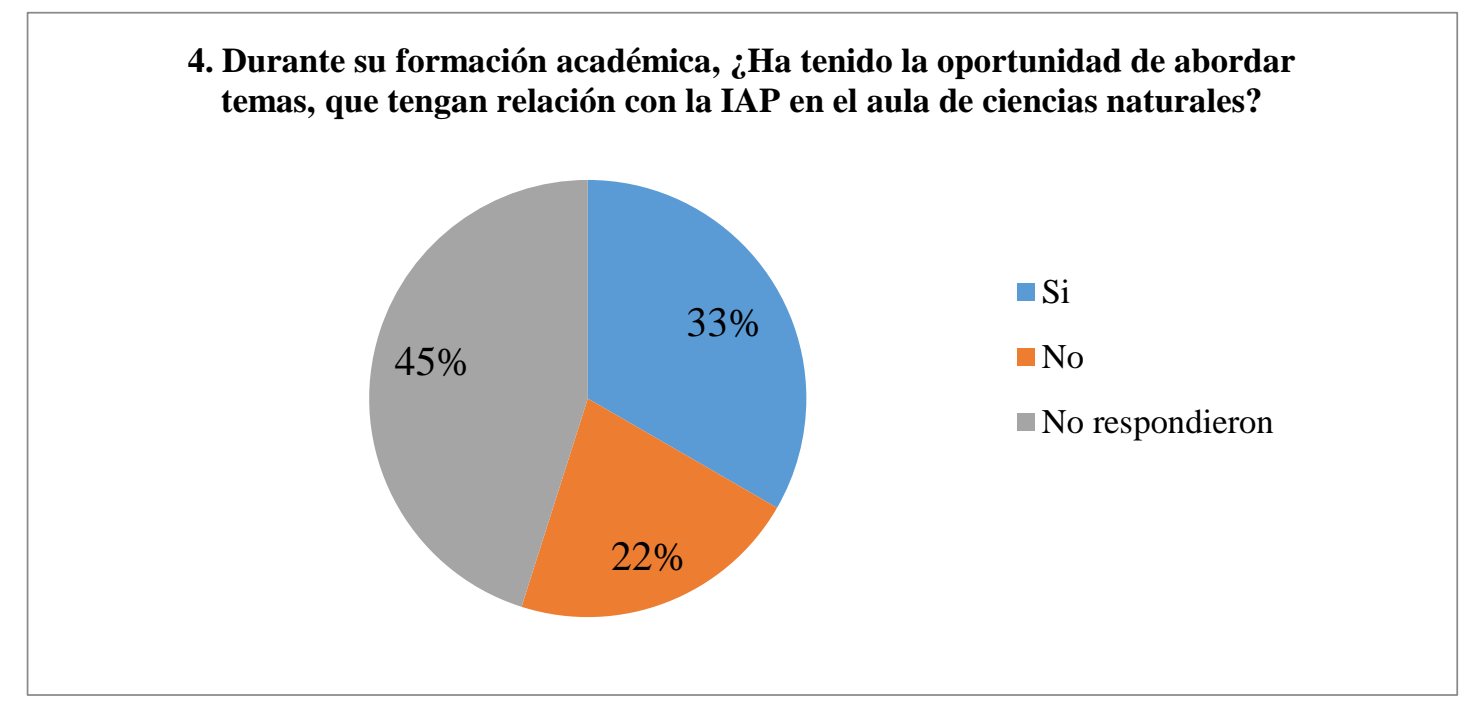

Figura 3: Pregunta 4. Fuente: Elaboración propia 
Tabla 3

Pregunta 4 de la encuesta. Porcentaje y cantidad de estudiantes

\begin{tabular}{lccc}
\hline OPCIÓN & SI & NO & No respondieron \\
Cantidad de estudiantes & 11 & 11 & 23 \\
Porcentaje \% & 33 & 22 & 45 \\
\hline
\end{tabular}

Fuente: Elaboración propia

En esta pregunta 4, la moda entre las personas encuestadas fue SI con un 33\%. Aunque, es poco representativa debido a que su porcentaje no sobrepasa la mitad de los estudiantes. Algunos estudiantes asociaban la IAP en el aula de ciencias naturales porque unos profesores hacían experimentos o tareas que se socializaban haciendo énfasis en el método científico; otros decían que la docente proponía parcialmente la IAP.

5. ¿El docente de ciencias naturales ha tenido la oportunidad de incluir alguna actividad de IAP en el aula en la cual usted haya participado?

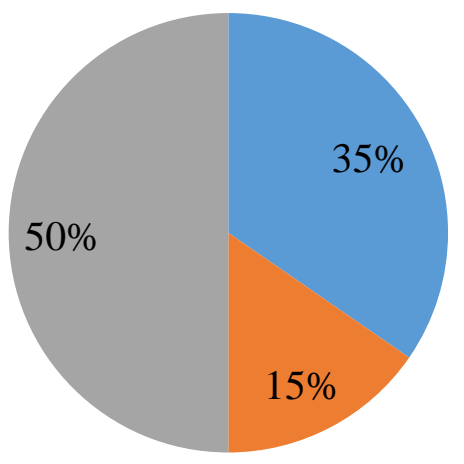

Figura 4: Pregunta 5. Fuente: Elaboración propia 
Tabla 4

Pregunta 5 de la encuesta. Porcentaje y cantidad de estudiantes

\begin{tabular}{lccc}
\hline OPCIÓN & SI & NO & No respondieron \\
Cantidad de estudiantes & 18 & 8 & 26 \\
Porcentaje \% & 35 & 15 & 50 \\
\hline
\end{tabular}

Fuente: Elaboración propia

En esta pregunta la moda entre las personas encuestadas fue SI con un 35\%, siendo esta poco representativa porque su porcentaje no sobrepasa la mitad del porcentaje. Las actividades que los estudiantes asociaban a la IAP que ha realizado con la profesora, eran experimentos sencillos de mezclas y métodos de separación de mezclas como el laboratorio de destilación simple.

6. ¿Cree importante incluir en la asignatura de ciencias naturales del Colegio Enrique Olaya Herrera, aspectos que fortalezcan la IAP en el aula?

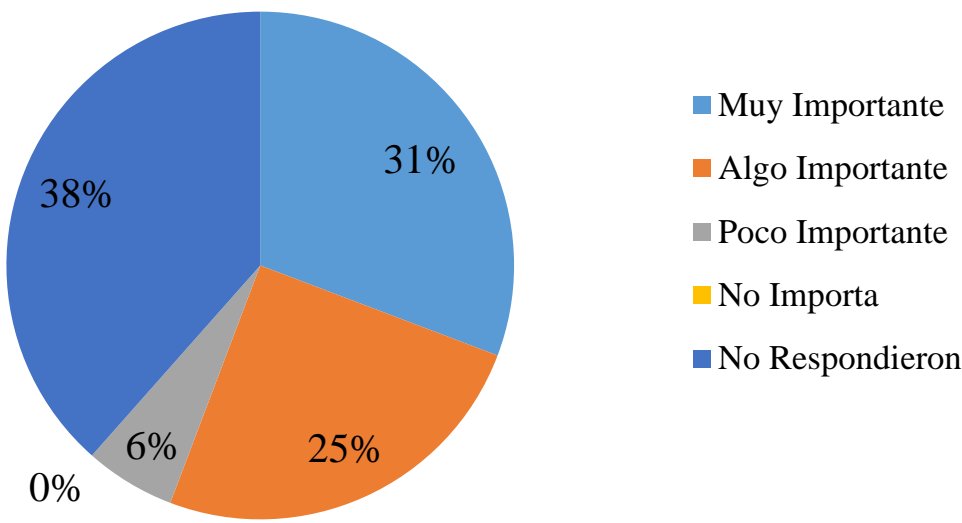

Figura 5: Pregunta 6. Fuente: Elaboración propia

Tabla 5

\begin{tabular}{lccccc}
\hline Pregunta 6 de la encuesta. Porcentaje y cantidad de estudiantes \\
\hline OPCIÓN & MI & AI & PI & NI & NR \\
Cantidad de estudiantes & 16 & 13 & 3 & 0 & 20 \\
Porcentaje \% & 31 & 25 & 6 & 0 & 38
\end{tabular}

Fuente: Elaboración propia 
En esta pregunta la novedad entre las personas encuestadas fue "muy importante" con un $31 \%$. Sin embargo, es poco representativa porque su porcentaje no sobrepasa la mitad de los estudiantes. Los estudiantes creían importante incluir aspectos que fortalezcan la IAP con la socialización de respuestas de consultas, participar activamente en clase e invitar a la comunidad aledaña al $\mathrm{CEOH}$ IED a ser partícipe de la clase. Otros exponían que la IAP serviría mucho para el aprendizaje de las ciencias naturales y que ayudaría a captar la atención del estudiante, además de impulsar a adquirir mayor conocimiento científico.

7. ¿Debe la comunidad participar en los procesos de IAP en el aula?

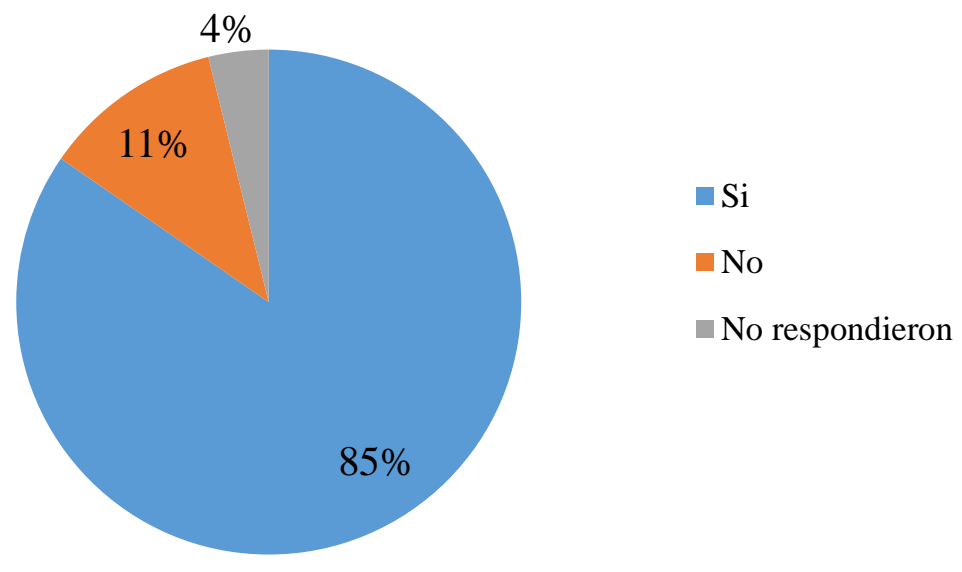

Figura 6: Pregunta 7. Fuente: Elaboración propia

Tabla 6

\begin{tabular}{lccc} 
Pregunta 7 de la encuesta. & Porcentaje y cantidad de estudiantes \\
\hline OPCIÓN & SI & NO & No respondieron \\
Cantidad de estudiantes & 44 & 6 & 2 \\
Porcentaje $\%$ & 85 & 12 & 4 \\
\hline
\end{tabular}

Fuente: Elaboración propia

En esta pregunta la moda entre las personas encuestadas fue SI, es representativa con un $85 \%$ porque indica que los estudiantes pensaban que la comunidad debía participar en los procesos de IAP. Algunos creían que se entenderían mejor el conocimiento científico si se incluye la comunidad en el aula. Además de conocer distintos aspectos de la comunidad desde un punto de vista crítico, 
asimismo fortalecer y ampliar el conocimiento para el futuro y para formar seres humanos conscientes.

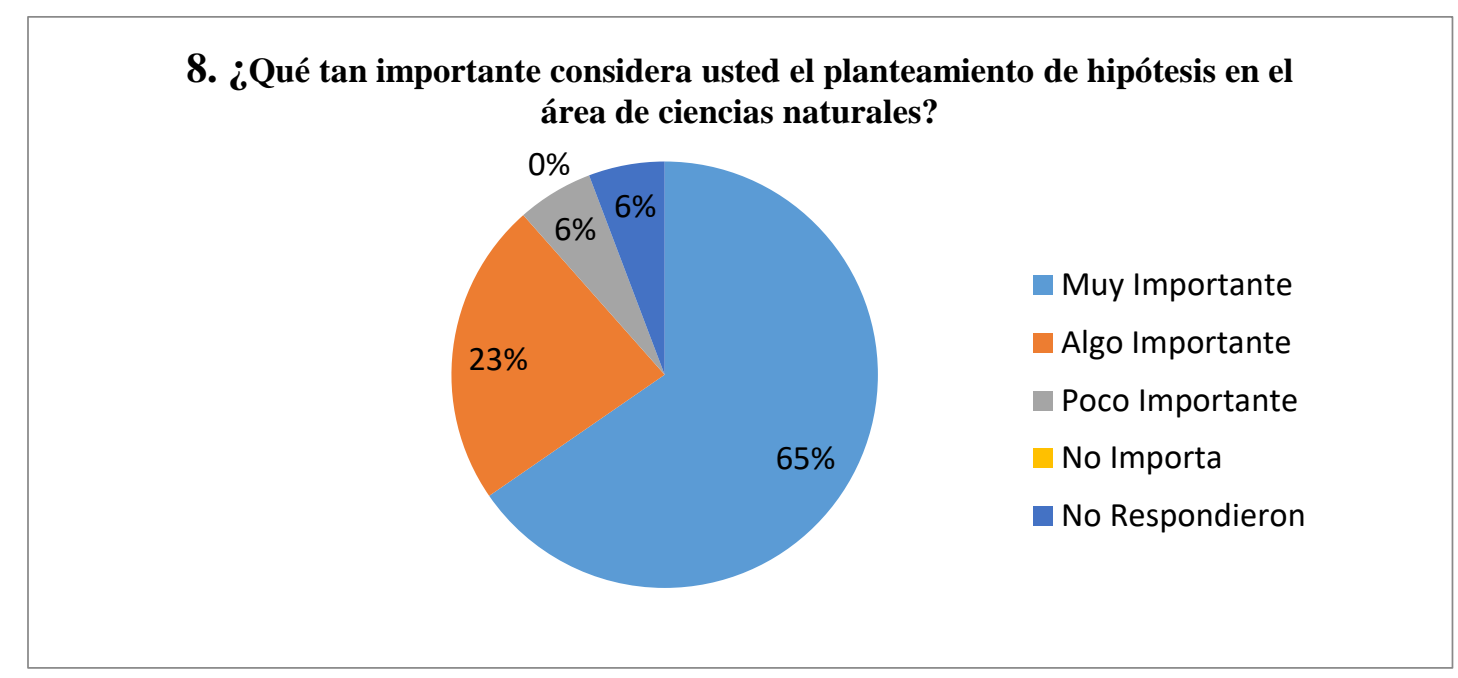

Figura 7: Pregunta 8. Fuente: Elaboración propia

Tabla 7

\begin{tabular}{lccccc}
\hline Pregunta 8 de la encuesta. Porcentaje y cantidad de estudiantes \\
\hline OPCIÓN & MI & AI & PI & NI & NR \\
Cantidad de estudiantes & 34 & 12 & 3 & 0 & 3 \\
Porcentaje \% & 65 & 23 & 6 & 0 & 6 \\
\hline
\end{tabular}

Fuente: Elaboración propia

En esta pregunta la moda entre las personas encuestadas fue "muy importante", es representativa con un $65 \%$ lo que indica que los estudiantes consideraban importante el planteamiento de hipótesis en el área de ciencias naturales.

Con el planteamiento de hipótesis, los estudiantes exponían que podrían tener algo de referencia y contrastar si es correcto o no un tópico para obtener un resultado sobre acontecimientos cotidianos donde se vea inmersa la IAP, también ayudan a resolver incógnitas al dar una respuesta a través de la experimentación, otros decían que sin hipótesis las ciencias naturales serían como una pared llena de ladrillos sin cemento. 
La encuesta realizada a los estudiantes constó de preguntas de selección múltiple con justificación de su respuesta, se concluye que los estudiantes no sabían el concepto de IAP, pero reconocían algunas características que se implementaba en el CEOH IED y en el área de ciencias naturales. Por otra parte, algunos estudiantes asociaban la IAP con apoyo de prácticas experimentales, aunque éste sólo es uno de tantos procesos que puede apoyar la IAP. Por ejemplo, en ciencias naturales los procesos de apoyo a la IAP son: el planteamiento de hipótesis, las consultas previas, actividades de integración y la participación de la comunidad; si bien en el aula de ciencias naturales esto se cumple parcialmente, faltaba fortalecer todo lo anterior para alcanzar un buen aprendizaje.

De igual manera, en este análisis se puede mencionar que los estudiantes consideraban importante incluir la IAP en el aula de ciencias naturales, esta se desenvolvería de una manera contextual y contemporánea, ayudaría a la socialización de las ciencias y por supuesto a hacer partícipe a la comunidad porque llevaría un proceso enriquecedor de alfabetización científica. Asimismo, creían importante discutir y reflexionar sobre lo que se realiza en el aula, es decir, crear un ambiente de colaboración entre el profesor, los estudiantes y la comunidad porque esta ayudaría a fortalecer la habilidad argumentativa y el conocimiento humano hacia el futuro.

\section{Etapa 3}

\section{ENTREVISTA}

Fecha: 1-noviembre-2013-Lugar: Colegio Enrique Olaya Herrera I.E.D.-Hora: 9 AM

Entrevistador(a): Jonathan Hernández Prieto Entrevistado(a): Profesora Titular de C. Naturales

\section{Introducción}

En el marco de las prácticas pedagógicas de los estudiantes del Departamento de Química de la Universidad Pedagógica Nacional, se desarrolla un proyecto de investigación llamado: "Determinación y propuesta de una investigación acción participativa en el aula de ciencias naturales del Colegio Enrique Olaya Herrera IED” Realizado por Jonathan Heiler Hernández Prieto 
Nota: La abreviatura IAP significa (Investigación-Acción-Participación), además, la entrevista esta parafraseada.

\section{Características de la entrevista}

Confidencialidad y no durara más de 20 minutos.

\section{Preguntas}

1. ¿Sabe usted, a qué hace referencia cuando se habla de IAP en el aula de ciencias naturales? Explique su respuesta

La IAP en el aula genera un proceso de investigación y de reflexión frente a los resultados que van obteniendo del proceso educativo, con el fin de mejorar las prácticas y generar estrategias que profundicen mejoramiento de la enseñanza de las ciencias naturales.

2. ¿Cree usted que tanto profesores como estudiantes pueden realizar IAP en el aula? ¿Por qué?

Claro, se puede generar IAP con ayuda del profesor. Sin embargo, debido a las condiciones que tienen la educación puede ser complicado, pero si se apoya se podría realizar.

3. ¿Supone usted que las prácticas experimentales se pueden utilizar como apoyo de la IAP? ¿Por qué?

$\mathrm{Si}$, se puede usar como instrumento de investigación para evaluar el saber hacer.

4. ¿Cree usted que es conveniente dejar consultas a los estudiantes para que las desarrollen en la casa? ¿Por qué?

Sí, son convenientes las consultas porque acercan a los estudiantes a una gran cantidad de conocimiento y que ellos tengan la habilidad de seleccionar cual de esos conocimientos es importante.

5. ¿Actualmente deja consultas? ¿Por qué?

$\mathrm{Si}$, dejo consultas para que ellos aprendan a seleccionar la información, pero antes de enviar la consulta les asesoro con respecto a qué es lo importante a seleccionar. 
6. ¿Considera que el planteamiento de hipótesis es necesario para apoyar los procesos de IAP en el aula? ¿Por qué?

Por supuesto son importantes porque les permite a los estudiantes un acercamiento al conocimiento científico mediante la predicción.

7. ¿Considera que la enseñanza de las ciencias basada en la indagación difiere de la enseñanza de las ciencias apoyada por la IAP? ¿Por qué?

No, tal vez no difiere la una de la otra mucho porque ambas son procesos de investigación.

8. ¿Supone que los estudiantes se sentirán más entusiasmados si trabajan y participan en los procesos de IAP? ¿Por qué?

Claro, cuando el estudiante hace parte del proceso activo, él se va sentir motivado en el proceso.

9. ¿Cree usted que la participación de la comunidad en los procesos de IAP enriquecería más las clases de ciencias naturales? ¿Por qué?

Sí, la enriquecería más porque está el contexto del estudiante dentro del aula. Entonces, esto permitiría que fuese más cercana la ciencia.

10. ¿Cree usted que la IAP ayudaría a estimular a los estudiantes para que sientan más interés por el área de ciencias naturales? ¿Por qué?

11 .

Sí, la participación en los procesos hace que el estudiante se sienta un ente activo e importante en el desarrollo del conocimiento y a veces puede romper la barrera de la enseñanza tradicional.

Posteriormente, para un análisis más detallado de todos los conceptos que se emplean en la entrevista y sus respetivas relaciones o asociaciones, se realizó la siguiente red conceptual: 
Esquema 2. Red Conceptual de Análisis de la Entrevista

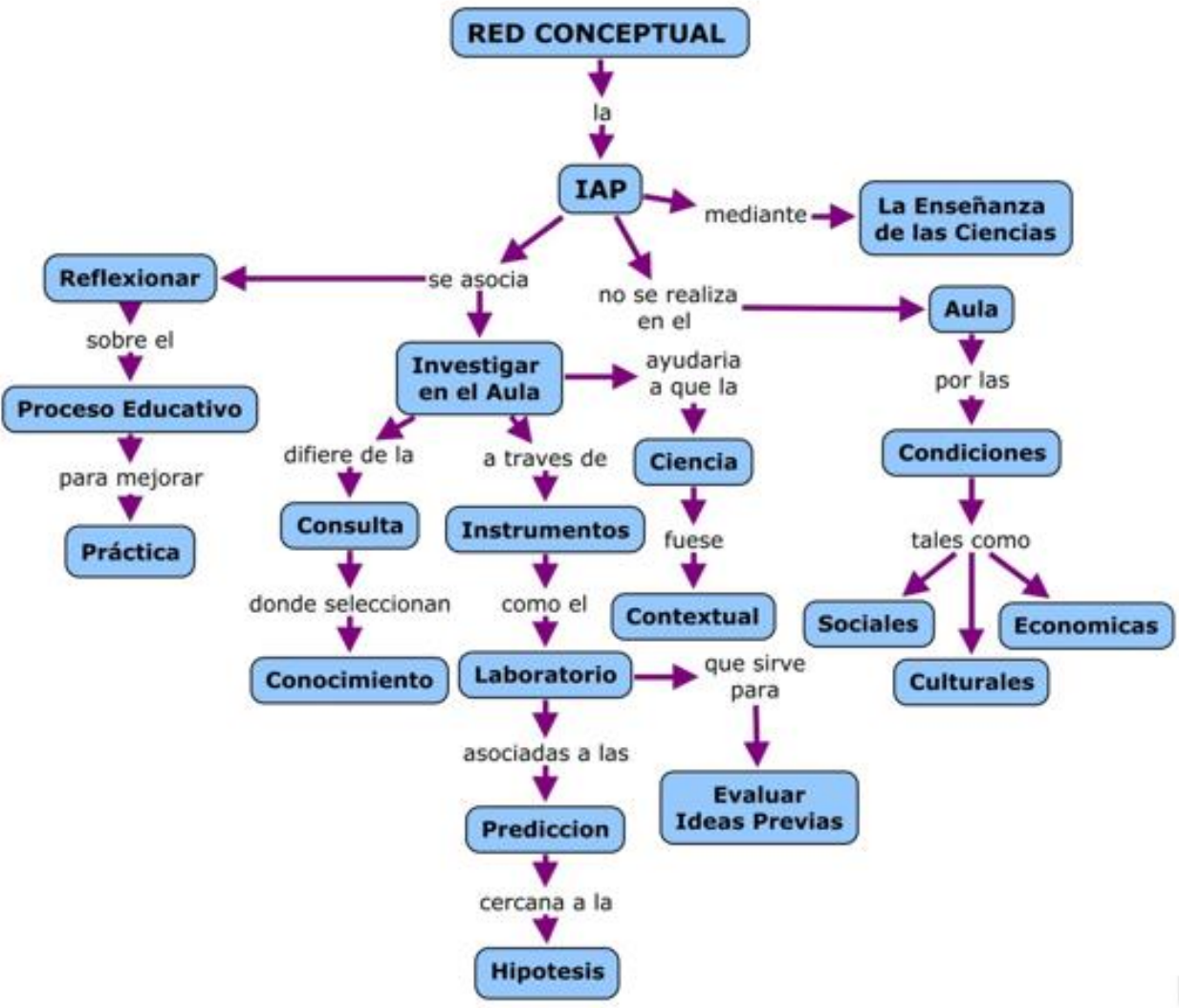

Fuente: Elaboración propia

En la entrevista, la profesora tiene un buen bagaje acerca de la metodología IAP y como se puede aplicar en el área de ciencias naturales. Sin embargo, considera este campo muy irreal porque para ella es difícil vincular ampliamente a la comunidad con las dinámicas sociales actuales. También, menciona que no solo debe colaborar la comunidad, sino que también los estudiantes deben participar activamente en el aula de ciencias naturales.

Por lo tanto, ella también ve viabilidad en la posible aplicación de la IAP, ya que el modelo pedagógico del CEOH IED es Constructivismo Social que impactaría positivamente los diferentes ámbitos que rodean la institución, tales como: el ámbito económico, social y cultural. Adicionalmente, cree que las prácticas experimentales y las hipótesis pueden ser uno de los 
principales apoyos para que se empiece a implementar de manera paulatina la metodología IAP, misma perspectiva que comparte este análisis.

Del mismo modo, reflexiona sobre la importancia de las consultas en el aula, ya que desde allí se puede dar una base sólida para empezar a realizar la IAP. Además, en la entrevista se habló del interés que generaría en los estudiantes la IAP en el aula y que según su opinión el estudiante se sentiría como sujeto activo en la clase de ciencias naturales, esto conlleva a afirmar que se está implementando de manera parcial en el CEOH IED la IAP. Por lo tanto, hay procesos inmersos en el aula que puede dar bases para la implementación de este estilo de investigación y este a su vez esté apoyado con la enseñanza de las ciencias, esto evidencia que se cumplió con el objetivo general del proyecto.

Desde los objetivos específicos se puede resaltar que las actividades de integración y las prácticas experimentales ayudan al estilo de investigación IAP. No obstante, falta incorporar más a la comunidad local con la institución, hay procesos parciales de IAP en el recinto de clase; estos resultados dan base para la construcción desde este estilo investigativo a una secuencia de actividades de agricultura urbana, plantas medicinales y suelos.

\section{Etapa 4}

Esquema 3: Secuencia de actividades

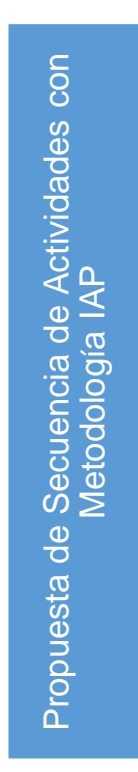

Actividad 1. Video explicativo y lectura de agricultura urbana de plantas aromáticas, suelos seguridad alimentaria

Actividad 2. Determinación de ph de suelos y selección de planta aromática a sembrar

Actividad 3. Protocolo de siembra en agricultura urbana junto a la familia

Actividad 4. Determinación de textura de suelo

Actividad 5. Estructuras químicas favorables a la salud de plantas aromáticas 
Es importante resaltar que esta propuesta no fue implementada. No obstante, queda como sugerencia en una segunda etapa de investigación donde se pueda implementar.

\section{Etapa 5}

Como se observa anteriormente etapa por etapa, el análisis se mostró después de los resultados y se realizó la respectiva triangulación.

\section{Conclusiones}

- Las prácticas experimentales pueden apoyar la metodología IAP para la enseñanza de las ciencias naturales. Además, se encontró que se implementan actividades parciales asociadas a esta metodología y al mismo tiempo, podían ayudar a mejorar la enseñanza de las ciencias naturales en el CEOH IED.

- Para vincular de una manera completa la IAP con el aula de ciencias naturales se debe actuar frente a situaciones problemáticas de la comunidad que requieren una respuesta y apoyo de las instituciones educativas.

- Los instrumentos empleados en este proyecto permitieron realizar una triangulación en el análisis, en el cual se evidencia una implementación parcial de la IAP.

- La metodología de IAP para algunos profesores del área de ciencias naturales puede ser algo irreal. Aunque, en los últimos años está tomando mucha fuerza y con el tiempo esta metodología empieza a afrontar situaciones problemáticas del contexto.

- La IAP en el marco de la Práctica Pedagógica del Departamento de Química de la Universidad Pedagógica Nacional, a futuro tendrá una visión integradora de comunidades y servirá de apoyo para muchos profesores en formación, igualmente para los actuales profesores que buscan cada día modificar su forma de investigar y enseñar, así como a la vez transformar la realidad de esas comunidades educativas. 


\section{Referencias Bibliográficas}

Borda, O. \& Erazo, M. (2010). Concepciones sobre ciencia e investigación en profesores de química en formación inicial: un estudio en el contexto de los trabajos experimentales. Rev. TED, 28, 41-56

Eizagirre, M. \& Zabala, N. (2000) Diccionario de Acción Humanitaria y Cooperación al Desarrollo. Recuperdado de: http://www.dicc.hegoa.ehu.es/listar/mostrar/132

Miranda, J. (2008). Modelo mediacional de aprendizaje situado para investigación educativa. Rev. Nod.y Nud., 25, 13-24

Moncayo, V. (2015). Una sociología sentipensante para América Latina / Orlando Fals Borda; antología y presentación. Buenos Aires: Clacso: Siglo XXI Editores. Recuperado de: http://biblioteca.clacso.edu.ar/clacso/se/20151027053622/AntologiaFalsBorda.pdf

Muñoz, J. (2005). Como desarrollar Competencias Investigativas En Educación, Colombia: Editorial Aula Abierta.

Navarro, A. (2005). La investigación social y educativa: un puente entre la escuela y su entorno. Rev. Nod.y Nud., 19, 67-74

Nistal, T. (2007). Ponencia Investigación-Acción Participativa y Mapas Sociales. U. Jaen, Depto de Psicología, p. 1-27. Recuperado de: http://comprenderparticipando.com/wpcontent/uploads/2016/04/Tomas-Alberich-Nistal-Investigacion-accion-participatival.pdf

Téllez, A. (2007). La Investigación Antropológica. España: Editorial Club Universitario.

\section{Agradecimientos.}

A mi familia y en especial a mi hermana, Carmen Hernández, por apoyarme e inspirarme; a la comunidad educativa del Colegio Enrique Olaya Herrera IED, a las orientaciones dadas por la profesora Margarita Rendón Fernández y la profesora Nancy Ortiz. 
